MATEMATIKA, 2017, Volume 33, Number 2, 215-226

(C) Penerbit UTM Press. All rights reserved

\title{
Block backward differentiation formulas for solving second order fuzzy differential equations
}

\author{
${ }^{1}$ Tiaw Kah Fookand and ${ }^{2}$ Zarina Bibi Ibrahim \\ Institute for Mathematical Research, Department of Mathematics \\ Faculty of Science, Universiti Putra Malaysia \\ 43400 Serdang, Selangor, Malaysia \\ e-mail: ${ }^{1}$ kelvin9175@yahoo.com, ${ }^{2}$ zarinabb@science.upm.edu.my
}

\begin{abstract}
In this paper, we study the numerical method for solving second order Fuzzy Differential Equations (FDEs) using Block Backward Differential Formulas (BBDF) under generalized concept of higher-order fuzzy differentiability. Implementation of the method using Newton iteration is discussed. Numerical results obtained by BBDF are presented and compared with Backward Differential Formulas (BDF) and exact solutions. Several numerical examples are provided to illustrate our methods.
\end{abstract}

Keywords Fuzzy differential equations; block backward differentiation formula; block differential formula; generalized differentiability.

AMS mathematics subject classification 65D05; 65D15; 65M15.

\section{Introduction}

The study of FDEs has been the interest of many researchers in recent years due to the suitability in modeling of real world problem for processing vague and subjective information. The concept of a fuzzy derivative was first introduced by Chang and Zadeh [1]. It was followed by Dubois and Prade [2], who used the extension principle in their approach. The first approach to solve FDEs is based on Zadeh's extension principle. In this approach the associated crisp second-order problem is solved in the obtained solution, the boundary values are substituted instead of the real constant. Another approach has been proposed by Puri and Ralescu [3], they generalized and extended the concept of Hukuhara differentiability ( $H$-derivative) from set-valued mappings to the class of fuzzy mappings. Then, fuzzy differential equation and initial value problems were extensively studied by Kaleva [4, 5] and by Seikkala [6]. A variety of exact, approximate and purely numerical methods are available to find the solution of a fuzzy initial value problem (FIVP). Some authors of the research papers have pursued methods based on the generalized derivative. Hence, we use this generalized derivative concept in the present paper.

In the last few years, second-order fuzzy differential equations [7-12] have been studied. There is a few investigations with different numerical methods have been devoted to the numerical solution of second-order fuzzy differential equations. In the paper [10] of Allahviranloo et al., the authors obtained the approximate solution of nth-order linear differential equations with fuzzy initial conditions by using the collocation method. Wang and Guo have developed numerical methods for addressing second-order fuzzy differential equation by Adomian decomposition method [11]. Meanwhile Rabiei et al. have developed the Fuzzy Improved Runge-Kutta Nystrom (FIRKN) method for solving second-order fuzzy differential equations [12]. In this paper, we want to propose block backward differentiation formula method for solving these equations. Therefore, this process would be developed through four sections. 
The paper is organized as follows. In Section 2, we give some basic definitions and theorem on FDEs. In Section 3, we show how Fuzzy version of BBDF is constructed. In Section 4, the numerical examples are provided to illustrate the validity and applicability of the method. Finally, some conclusions are given.

\section{Preliminaries}

We introduce the necessary notation and give some definitions which will be used throughout the paper; see [13-15].

We consider all real numbers $\mathbb{R}$. A fuzzy number is a mapping $u: \mathbb{R} \rightarrow[0,1]$ with the following properties:

(a) $u$ is normal, there exists an $x_{0} \in \mathbb{R}$ for which $u\left(x_{0}\right)=1$,

(b) $u$ is upper semi-continuous,

(c) $u$ is fuzzy convex, i.e., $u(\alpha x+(1-\alpha) y) \geq \min u(x), u(y)\}$ for all $x, y \in \mathbb{R}, \alpha \in[0,1]$,

(d) $\operatorname{supp} u=\{x \in \mathbb{R} \mid u(x)>0\}$ is the support of the $u$, and its closure cl(supp $u$ ) is compact.

Definition 1 An arbitrary fuzzy number $u$ in the parametric form is represented by an ordered pair of functions $(\underline{u} \bar{u})$ which satisfy the following requirements:

(i) $\bar{u}: \alpha \rightarrow \bar{u}^{\alpha} \in \mathbb{R}$ is a bounded left-continuous non decreasing function over $[0,1]$,

(ii) $\underline{u}: \alpha \rightarrow \underline{u}^{\alpha} \in \mathbb{R}$ is a bounded left-continuous non increasing function over $[0,1]$,

(iii) $\underline{u}^{\alpha} \leq \bar{u}^{\alpha}, 0 \leq \alpha \leq 1$.

Let $D: E \times E \rightarrow \mathbb{R}^{+} \cup\{0\}$ be defined by $D(u, v)=d\left([u]^{\alpha},[v]^{\alpha}\right)$ where $d$ is the Hausdorff metric defined in $(P(\mathbb{R}), d)$. Then $D$ is a metric on $E$. Further, $(E, D)$ is a complete metric space has the following properties:

(i) $D(u \oplus w, v \oplus w)=D(u, v)$, for all $u, v, w \in E$,

(ii) $D(k \odot u, k \odot v)=|k| D(u, v)$, for all $k \in \mathbb{R}, u, v \in E$,

(iii) $D(u \oplus v, w \oplus e) \leq D(u, w)+D(v, e)$ for all $u, v, w, e \in E$,

(iv) $(D, E)$ is a complete metric space.

Definition 2 Consider $x, y \in \mathbb{R}$. If there exist $z \in \mathbb{R}$ such that $x=y \ominus z$, then $z$ is called the H-difference of $x$ and $y$ and it is denoted by $x \ominus y$.

In this paper, the sign " $\ominus$ " always stands for H-difference and note that $x \ominus y \neq x+(-y)$.

Definition 3 Let $F: I \rightarrow \mathbb{R}$. For $t_{0} \in I$, we say $F$ is differentiable at $t_{0}$, if there exists an element $f^{\prime}\left(t_{0}\right) \in \mathbb{R}$ such that either

(i) for all $h>0$ sufficiently small, $\exists f\left(t_{0}+h\right) \ominus f\left(t_{0}\right), \exists f\left(t_{0}\right) \ominus f\left(t_{0}-h\right)$ and the limits (in the metric $d$ )

$$
\frac{f\left(t_{0}+h\right) \ominus f\left(t_{0}\right)}{h}=\frac{f\left(t_{0}\right) \ominus f\left(t_{0}-h\right)}{h}=f^{\prime}\left(t_{0}\right),
$$

(ii) for all $h>0$ sufficiently small, $\exists f\left(t_{0}\right) \ominus f\left(t_{0}+h\right), \exists f\left(t_{0}-h\right) \ominus f\left(t_{0}\right)$ and the limits (in the metric d)

$$
\frac{f\left(t_{0}\right) \ominus f\left(t_{0}+h\right)}{-h}=\frac{f\left(t_{0}-h\right) \ominus f\left(t_{0}=f^{\prime}\left(t_{0}\right)\right)}{-h},
$$


(iii) for all $h>0$ sufficiently small, $\exists f\left(t_{0}+h\right) \ominus f\left(t_{0}\right), \exists f\left(t_{0}-h\right) \ominus f\left(t_{0}\right)$ and the limits (in the metric $d$ )

$$
\frac{f\left(t_{0}+h\right) \ominus f\left(t_{0}\right)}{h}=\frac{f\left(t_{0}-h\right) \ominus f\left(t_{0}=f^{\prime}\left(t_{0}\right)\right)}{-h},
$$

(iv) for all $h>0$ sufficiently small, $\exists f\left(t_{0}\right) \ominus f\left(t_{0}+h\right), \exists f\left(t_{0}\right) \ominus f\left(t_{0}-h\right)$ and the limits (in the metric d)

$$
\frac{f\left(t_{0}\right) \ominus f\left(t_{0}+h\right)}{-h}=\frac{f\left(t_{0}\right) \ominus f\left(t_{0}-h=f^{\prime}\left(t_{0}\right)\right)}{h} .
$$

The fuzzy set $f\left(t_{0}\right)$ is called the hukuhara derivative of $f$ at $t_{0}$.

In [16], the authors consider four cases for derivatives. Here we only consider the two first cases of Definition 3. In the other cases, the derivative is trivial because it is reduced to a crisp element. We say $f$ is $(1)$-differentiable on $(a, b)$ if $f$ is differentiable with the meaning (i) of Definition 3 and also (2) - differentiable that $f$ satisfies in the Definition 4 case (ii).

Theorem $1[17]$ Let $f:(a, b) \rightarrow E$ be a function and denote $[F(t)]^{r}=\left[f_{r}(t), g_{r}(t)\right]$, for each $r \in[0,1]$. Then

(i) if $f$ is $(1)$ - differentiable, then $f_{r}(t)$ and $g_{r}(t)$ are differentiable functions and

$$
\left[F^{\prime}(t)\right]^{r}=\left[f_{r}^{\prime}(t), g_{r}^{\prime}(t)\right],
$$

(ii) if fis $(2)$ - differentiable, then $f_{r}(t)$ and $g_{r}(t)$ are differentiable functions and

$$
\left[F^{\prime}(t)\right]^{r}=\left[g_{r}^{\prime}(t), f_{r}^{\prime}(t)\right] .
$$

Consider the second-order fuzzy initial value problem:

$$
\left\{\begin{array}{l}
y^{\prime \prime}=f\left(t, y, y^{\prime}\right), t \in\left[t_{0}, T\right] \\
y\left(t_{0}\right)=y_{0}, y^{\prime}(0)=y^{\prime}{ }_{0}
\end{array}\right.
$$

where $f$ is a fuzzy function with $r$-level sets of initial values

$$
\left[y_{0}^{\prime}\right]_{r}=\left[y_{1}^{\prime}(0 ; r), y_{2}^{\prime}(0 ; r)\right], r \in[0,1] .
$$

We write

$$
y(t, y)=\left[y_{1}(t ; r), y_{2}(t ; r)\right], y^{\prime}(t, y)=\left[y_{1}^{\prime}(t ; r), y_{2}^{\prime}(t ; r)\right] \text { and } f(t, y)=\left[f_{1}(t, y), f_{2}(t, y)\right] \text {, }
$$

where

$$
\begin{aligned}
& f_{1}(t, y)=F\left[t, y_{1}(t ; r), y_{2}(t ; r)\right], \\
& f_{2}(t, y)=G\left[t, y_{1}(t ; r), y_{2}(t ; r)\right] .
\end{aligned}
$$

By using the extension principle, when $y(t)$ is a fuzzy number we have the membership function

$$
f(t, y(t))(s)=\sup \{y(t)(\tau) \mid s=f(t, \tau)\}, s \in R .
$$


It follows that

$$
[f(t, y)]^{r}=\left[f_{1}(t, y ; r), f_{2}(t, y ; r)\right], r \in[0,1]
$$

where

$$
\begin{aligned}
& f_{1}(t, y ; r)=\min \left\{f(t, u) \mid u \in\left[y_{1}(r), y_{2}(r)\right]\right\}, \\
& f_{2}(t, y ; r)=\max \left\{f(t, u) \mid u \in\left[y_{1}(r), y_{2}(r)\right]\right\} .
\end{aligned}
$$

Definition 4 [18] Let $f:\left(t_{0}, T\right) \times E \rightarrow E$ and $t_{0} \in\left(t_{0}, T\right)$. We say that $f$ is strongly generalized differentiable of the second order at $t_{0}$, if there exists an element $f^{\prime \prime}\left(t_{0}\right) \in E$, such that

(i) for all $h>0$ sufficiently small, $\exists f^{\prime}\left(t_{0}+h\right) \ominus f^{\prime}\left(t_{0}\right), \exists f^{\prime}\left(t_{0}\right) \ominus f^{\prime}\left(t_{0}-h\right)$ and the limits (in the metric D)

$$
\begin{gathered}
\frac{f^{\prime}\left(t_{0}+h\right) \ominus f^{\prime}\left(t_{0}\right)}{h}=\frac{f^{\prime}\left(t_{0}\right) \ominus f^{\prime}\left(t_{0}-h\right)}{h} \\
=f^{\prime \prime}\left(t_{0}\right)
\end{gathered}
$$

or (ii) for all $h>0$ sufficiently small, $\exists f^{\prime}\left(t_{0}\right) \ominus f^{\prime}\left(t_{0}+h\right), \exists f^{\prime}\left(t_{0}-h\right) \ominus f^{\prime}\left(t_{0}\right)$ and the limits (in the metric D)

$$
\frac{f^{\prime}\left(t_{0}\right) \ominus f^{\prime}\left(t_{0}+h\right)}{-h}=\frac{f^{\prime}\left(t_{0}-h\right) \ominus f^{\prime}\left(t_{0}\right)}{-h}=f^{\prime \prime}\left(t_{0}\right)
$$

or (iii) for all $h>0$ sufficiently small, $\exists f^{\prime}\left(t_{0}+h\right) \ominus f^{\prime}\left(t_{0}\right), \exists f^{\prime}\left(t_{0}-h\right) \ominus f^{\prime}\left(t_{0}\right)$ and the limits (in the metric D)

$$
\frac{f^{\prime}\left(t_{0}+h\right) \ominus f^{\prime}\left(t_{0}\right)}{h}=\frac{f^{\prime}\left(t_{0}-h\right) \ominus f^{\prime}\left(t_{0}\right)}{-h}=f^{\prime \prime}\left(t_{0}\right)
$$

or (iv) for all $h>0$ sufficiently small, $\exists f^{\prime}\left(t_{0}\right) \ominus f^{\prime}\left(t_{0}+h\right), \exists f^{\prime}\left(t_{0}\right) \ominus f^{\prime}\left(t_{0}-h\right)$ and the limits (in the metric D)

$$
\frac{f^{\prime}\left(t_{0}\right) \ominus f\left(t_{0}+h\right)}{-h}=\frac{f^{\prime}\left(t_{0}\right) \ominus f\left(t_{0}-h\right)}{h}=f^{\prime \prime}\left(t_{0}\right) .
$$

For a supposed fuzzy function $f$, one has two possibilities, according to definitions 3 and 4 , to obtain the derivative of $f$ over $t: \underline{f}(t)$ and $\bar{f}(t)$. Then for each of these two derivatives, one has again two possibilities:

$$
D_{1}^{(1)}\left(D_{1}^{(1)} f(t), D_{2}^{(1)} f(t)\right) \text { and } D_{1}^{(1)}\left(D_{2}^{(1)} f(t)\right), D_{2}^{(1)}\left(D_{2}^{(1)} f(t)\right),
$$

respectively.

\section{Review the formulation of block backward differentiation formu- las method}

In this paper, we review the formulation of $\operatorname{BBDF}$ proposed by Ibrahim $[19,20]$. The BBDF is modified into a fuzzy version form to solve the second order FDEs in the form 
of equation in (7). To derive the formula of BBDF, Lagrange interpolating polynomial $P_{k}(x)$ is used to interpolate the values $y_{n}, y_{n-1}, \ldots, y_{n-k+1}$ at the interpolation points $x_{n}$, $x_{n-1}, \ldots, x_{n-k+1}$, in term of a Lagrange polynomial was defined as follows,

$$
P_{k}(x)=\sum_{j=0}^{k} L_{k, j}(x) y\left(x_{n+1-j}\right)
$$

where

$$
L_{k, j}(x)=\prod_{\substack{i=0 \\ i \neq j}}^{k} \frac{\left(x-x_{n+1-i}\right)}{\left(x_{n+1-j}-x_{n+1-i}\right)}, \text { for each } j=0,1, \ldots, k .
$$

In order to obtain the equations, the backvalues of $y_{n+2-i}, i=0,1, \ldots, 4$, are used to interpolate the Lagrange polynomial. From the equation in $(7),\left[t_{0}, t_{n}\right]$ is divided into five points $x_{n-2}, x_{n-1}, x_{n}, x_{n+1}, x_{n+2}$. Let $h$ is the constant step size, the equation obtains from the Lagrange polynomial are as below,

$$
\begin{aligned}
P(x) & =\frac{\left(x-x_{n-2}\right)\left(x-x_{n-1}\right)\left(x-x_{n}\right)\left(x-x_{n+1}\right)}{\left(x_{n+2}-x_{n-2}\right)\left(x_{n+2}-x_{n-1}\right)\left(x_{n+2}-x_{n}\right)\left(x_{n+2}-x_{n+1}\right)} y\left(x_{n+2}\right) \\
& +\frac{\left(x-x_{n-2}\right)\left(x-x_{n-1}\right)\left(x-x_{n}\right)\left(x-x_{n+2}\right)}{\left(x_{n+1}-x_{n-2}\right)\left(x_{n+1}-x_{n-1}\right)\left(x_{n+1}-x_{n}\right)\left(x_{n+1}-x_{n+2}\right)} y\left(x_{n+1}\right) \\
& +\frac{\left(x-x_{n-2}\right)\left(x-x_{n-1}\right)\left(x-x_{n+1}\right)\left(x-x_{n+2}\right)}{\left(x_{n}-x_{n-2}\right)\left(x_{n}-x_{n-1}\right)\left(x_{n}-x_{n+1}\right)\left(x_{n}-x_{n+2}\right)} y\left(x_{n}\right) \\
& +\frac{\left(x-x_{n-2}\right)\left(x-x_{n}\right)\left(x-x_{n+1}\right)\left(x-x_{n+2}\right)}{\left(x_{n-1}-x_{n-2}\right)\left(x_{n-1}-x_{n}\right)\left(x_{n-1}-x_{n+1}\right)\left(x_{n-1}-x_{n+2}\right)} y\left(x_{n-1}\right) \\
& +\frac{\left(x-x_{n-1}\right)\left(x-x_{n}\right)\left(x-x_{n+1}\right)\left(x-x_{n+2}\right)}{\left(x_{n-2}-x_{n-1}\right)\left(x_{n-2}-x_{n}\right)\left(x_{n-2}-x_{n+1}\right)\left(x_{n-2}-x_{n+2}\right)} y\left(x_{n-1}\right) .
\end{aligned}
$$

Substituting

$$
s=\frac{x-x_{n+1}}{h} \Rightarrow x=x_{n+1}+s h
$$

into Equation (15), then differentiate once at $x=x_{n+1}$ with respect to $s$ and substitute $s=0$ gives

$$
y_{n+1}^{\prime}=\frac{1}{h}\left(-\frac{1}{12} y_{n-2}+\frac{1}{2} y_{n-1}-\frac{3}{2} y_{n}+\frac{5}{6} y_{n+1}+\frac{1}{4} y_{n+2}\right) .
$$

Similarly, differentiating Equation (15) once at $x=x_{n+2}$ and substituting $s=1$, gives

$$
y_{n+2}^{\prime}=\frac{1}{h}\left(\frac{1}{4} y_{n-2}-\frac{4}{3} y_{n-1}+3 y_{n}-4 y_{n+1}+\frac{25}{12} y_{n+2}\right) .
$$

Then, differentiate twice the interpolating polynomial in Equation (15), evaluate at $x=$ $x_{n+2}$ and substitute $s=0$ gives

$$
y_{n+1}=-\frac{1}{20} y_{n-2}+\frac{1}{5} y_{n-1}+\frac{3}{10} y_{n}+\frac{11}{20} y_{n+2}-\frac{3}{5} h^{2} f_{n+1} .
$$


Differentiate twice the interpolating polynomial in (15) at $x=x_{n+2}$ and substitute $s=1$ gives the formula for second point,

$$
y_{n+2}=-\frac{11}{35} y_{n-2}+\frac{8}{5} ? 6_{n-1}-\frac{114}{35} y_{n}+\frac{104}{35} y_{n+1}+\frac{12}{35} h^{2} f_{n+2} .
$$

\section{Implementation of block backward differentiation formulas (BBDF) in fuzzy version}

In this section, the BBDF formula is modified into a fuzzy version of BBDF (FBBDF) to suit for the second order FDEs problem. Consider the second-order fuzzy initial value problem in the Equation (7). Let $Y(t ; r)=[\underline{Y}(t ; r), \bar{Y}(t ; r)]$ be the exact solution and $y(t ; r)=[\underline{y}(t ; r), \bar{y}(t ; r)]$ be the approximate solution where $r \in[0,1]$ and $t \in[0, T]$.

Equation (16-19) is modified into FBBDF and gives the following

$$
\begin{aligned}
& \left\{\begin{array}{l}
\underline{y}_{n+1}^{\prime}=\frac{1}{h}\left(-\frac{1}{12} \underline{y}_{n-2}+\frac{1}{2} \underline{y}_{n-1}-\frac{3}{2} \underline{y}_{n}+\frac{5}{6} \underline{y}_{n+1}+\frac{1}{4} \underline{y}_{n+2}\right) \\
\underline{y}_{n+1}=-\frac{1}{20} \underline{y}_{n-2}+\frac{1}{5} \underline{y}_{n-1}+\frac{3}{10} \underline{y}_{n}+\frac{11}{20} \underline{y}_{n+2}-\frac{3}{5} h^{2} \underline{f}_{n+1}[t, \underline{y}(t, r), \bar{y}(t, r)] \\
\underline{y}_{n+2}^{\prime}=\frac{1}{h}\left(\frac{1}{4} \underline{y}_{n-2}-\frac{4}{3} \underline{y}_{n-1}+3 \underline{y}_{n}-4 \underline{y}_{n+1}+\frac{25}{12} \underline{y}_{n+2}\right) \\
\underline{y}_{n+2}=-\frac{11}{35} \underline{y}_{n-2}+\frac{8}{5} \underline{y}_{n-1}-\frac{114}{35} \underline{y}_{n}+\frac{104}{35} \underline{y}_{n+1}+\frac{12}{35} h^{2} \underline{f}_{n+2}[t, \underline{y}(t, r), \bar{y}(t, r)]
\end{array}\right. \\
& \left\{\begin{array}{l}
\bar{y}_{n+1}^{\prime}=\frac{1}{h}\left(-\frac{1}{12} \bar{y}_{n-2}+\frac{1}{2} \bar{y}_{n-1}-\frac{3}{2} \bar{y}_{n}+\frac{5}{6} \bar{y}_{n+1}+\frac{1}{4} \bar{y}_{n+2}\right) \\
\bar{y}_{n+1}=-\frac{1}{20} \bar{y}_{n-2}+\frac{1}{5} \bar{y}_{n-1}+\frac{3}{10} \bar{y}_{n}+\frac{11}{20} \bar{y}_{n+2}-\frac{3}{5} h^{2} \bar{f}_{n+1}[t, \underline{y}(t, r), \bar{y}(t, r)] \\
\bar{y}_{n+2}^{\prime}=\frac{1}{h}\left(\frac{1}{4} \bar{y}_{n-2}-\frac{4}{3} \bar{y}_{n-1}+3 \bar{y}_{n}-4 \bar{y}_{n+1}+\frac{25}{12} \bar{y}_{n+2}\right) \\
\bar{y}_{n+2}=-\frac{11}{35} \bar{y}_{n-2}+\frac{8}{5} \bar{y}_{n-1}-\frac{114}{35} \bar{y}_{n}+\frac{104}{35} \bar{y}_{n+1}+\frac{12}{35} h^{2} \bar{f}_{n+2}[t, \underline{y}(t, r), \bar{y}(t, r)]
\end{array}\right.
\end{aligned}
$$

The following computations are carried out to obtain the approximations:

(i) Compute $Y_{n+1, n+2}^{(i)}$ and $Y_{n+1, n+2}^{(i)}$ using the predictor formula.

(ii) Solved $E_{n+1, n+2}^{(i+1)}$ and followed by ${E^{\prime}}_{n+1, n+2}^{(i+1)}$ for the corresponding linear system.

(iii) Computed the corrected value of $Y_{n+1, n+2}^{(i)}$ and $Y_{n+1, n+2}^{\prime(i)}$ with $E_{n+1, n+2}^{(i+1)}$ and $E_{n+1, n+2}^{(i+1)}$ respectively.

The notation $E$ is the error bound for the newton iteration.

\section{$5 \quad$ Numerical examples and discussion}

In this section, we solved the fuzzy initial value problems to show the accuracy of the proposed methods. The results of the exact and the approximate solutions are illustrated in the tables and figures. A comparison between the approximate solutions and the exact solutions is carried out to obtain the errors.

Let the exact solution be $Y(t ; r)=[\underline{Y}(t ; r), \bar{Y}(t ; r)]$. The absolute error formula, considered in Table 1-2, is as follows:

The error, $\varepsilon$ is defined as the maximum error through the whole interval of integration. Maximum Error $=\epsilon$,

$$
\underline{\varepsilon}=|\underline{y}-\underline{Y}|, \bar{\varepsilon}=|\bar{y}-\bar{Y}| .
$$


The notation used in the tables and figures take the following meaning:

$h:$ Step size

$r$ : Fuzzy numbers with fuzzy bounded $r$-level interval

$\underline{Y}$ : Lower bounded exact solution

$\bar{Y}$ : Upper bounded exact solution

$\underline{y}$ : Lower bounded approximate solution

$\bar{y}$ : Upper bounded approximate solution,

Example 1 Consider the following fuzzy linear initial value problem.

$$
\begin{aligned}
& y "(t)=-y(t), t \geq 0, \\
& y(0)=0, y^{\prime}(0)=[0.9+0.1 r, 1.1-0.1 r] .
\end{aligned}
$$

The exact solution at $t=1$ using (1)-differentiability is given by:

$$
Y(t ; r)=[(0.9+0.1 r) \sin (t)(1.1-0 . r) \sin (t)]
$$

Source: Allahviranloo et al. [9]

Table 1: Error at $t=1$ in solving problem 1

\begin{tabular}{|c|c|c|c|c|c|}
\hline \multicolumn{2}{|c|}{} & \multicolumn{2}{c|}{ BDF } & \multicolumn{2}{c|}{ BBDF } \\
\hline$h$ & $r$ & $\underline{\varepsilon}$ & $\bar{\varepsilon}$ & $\underline{\varepsilon}$ & $\bar{\varepsilon}$ \\
\hline \multirow{4}{*}{$10^{-1}$} & 0 & $3.09591 \mathrm{e}-05$ & $3.78389 \mathrm{e}-05$ & $5.40487 \mathrm{e}-05$ & $6.60595 \mathrm{e}-05$ \\
\cline { 2 - 6 } & 0.2 & $3.16471 \mathrm{e}-05$ & $3.71510 \mathrm{e}-05$ & $5.52498 \mathrm{e}-05$ & $6.48584 \mathrm{e}-05$ \\
\cline { 2 - 6 } & 0.4 & $3.23351 \mathrm{e}-05$ & $3.64630 \mathrm{e}-05$ & $5.64509 \mathrm{e}-05$ & $6.36573 \mathrm{e}-05$ \\
\cline { 2 - 6 } & 0.6 & $3.30231 \mathrm{e}-05$ & $3.57750 \mathrm{e}-05$ & $5.76519 \mathrm{e}-05$ & $6.24563 \mathrm{e}-05$ \\
\cline { 2 - 6 } & 0.8 & $3.37111 \mathrm{e}-05$ & $3.50870 \mathrm{e}-05$ & $5.88530 \mathrm{e}-05$ & $6.12552 \mathrm{e}-05$ \\
\cline { 2 - 6 } & 1.0 & $3.43990 \mathrm{e}-05$ & $3.43990 \mathrm{e}-05$ & $6.00541 \mathrm{e}-05$ & $6.00541 \mathrm{e}-05$ \\
\hline \multicolumn{2}{|c|}{ Execution time } & \multicolumn{3}{|c|}{$1.26 \mathrm{~s}$} & \multicolumn{3}{c|}{$0.6 \mathrm{~s}$} \\
\hline
\end{tabular}

\begin{tabular}{|c|c|c|c|c|c|}
\hline \multicolumn{2}{|c|}{} & \multicolumn{2}{c|}{ BDF } & \multicolumn{2}{c|}{ BBDF } \\
\hline$h$ & $r$ & $\underline{\varepsilon}$ & $\bar{\varepsilon}$ & $\underline{\varepsilon}$ & $\bar{\varepsilon}$ \\
\hline \multirow{4}{*}{$10^{-2}$} & 0 & $3.14945 \mathrm{e}-08$ & $3.84933 \mathrm{e}-08$ & $6.85061 \mathrm{e}-08$ & $8.37298 \mathrm{e}-08$ \\
\cline { 2 - 7 } & 0.2 & $3.21944 \mathrm{e}-08$ & $3.77934 \mathrm{e}-08$ & $7.00285 \mathrm{e}-08$ & $8.22074 \mathrm{e}-08$ \\
\cline { 2 - 7 } & 0.4 & $3.28943 \mathrm{e}-08$ & $3.70935 \mathrm{e}-08$ & $7.15509 \mathrm{e}-08$ & $8.06850 \mathrm{e}-08$ \\
\cline { 2 - 7 } & 0.6 & $3.35941 \mathrm{e}-08$ & $3.63937 \mathrm{e}-08$ & $7.30733 \mathrm{e}-08$ & $7.91627 \mathrm{e}-08$ \\
\cline { 2 - 7 } & 0.8 & $3.42940 \mathrm{e}-08$ & $3.56938 \mathrm{e}-08$ & $7.45956 \mathrm{e}-08$ & $7.76403 \mathrm{e}-08$ \\
\cline { 2 - 7 } & 1.0 & $3.49939 \mathrm{e}-08$ & $3.49939 \mathrm{e}-08$ & $7.61179 \mathrm{e}-08$ & $7.61179 \mathrm{e}-08$ \\
\hline \multicolumn{2}{|c|}{$1.84 \mathrm{~s}$} & \multicolumn{3}{c|}{$0.9 \mathrm{~s}$} \\
\hline
\end{tabular}




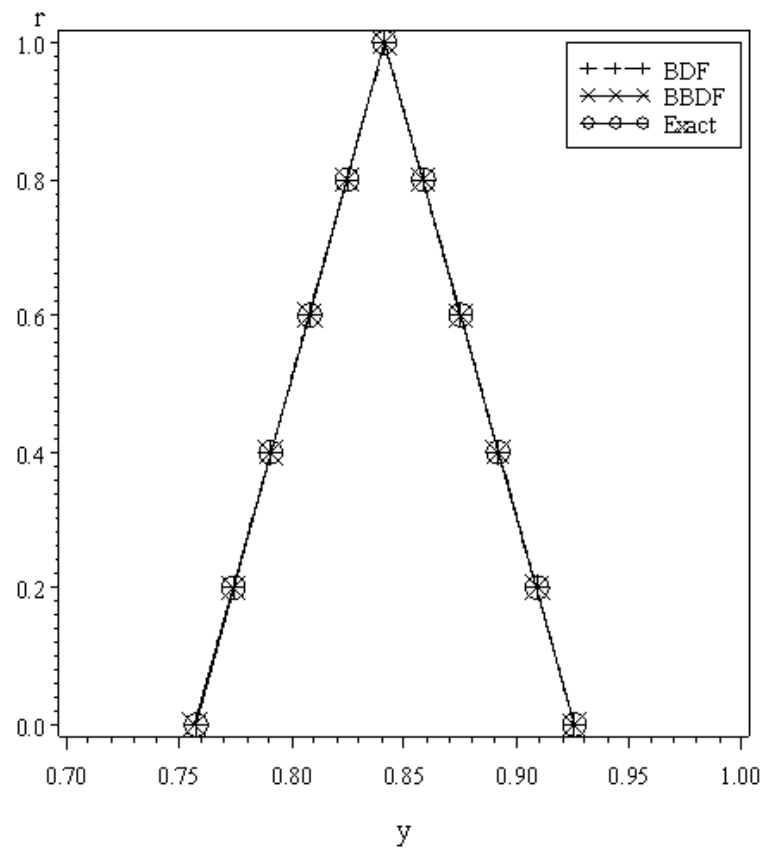

Figure 1: The exact solutions and the approximate solutions in Table 1 with $h=0.1$

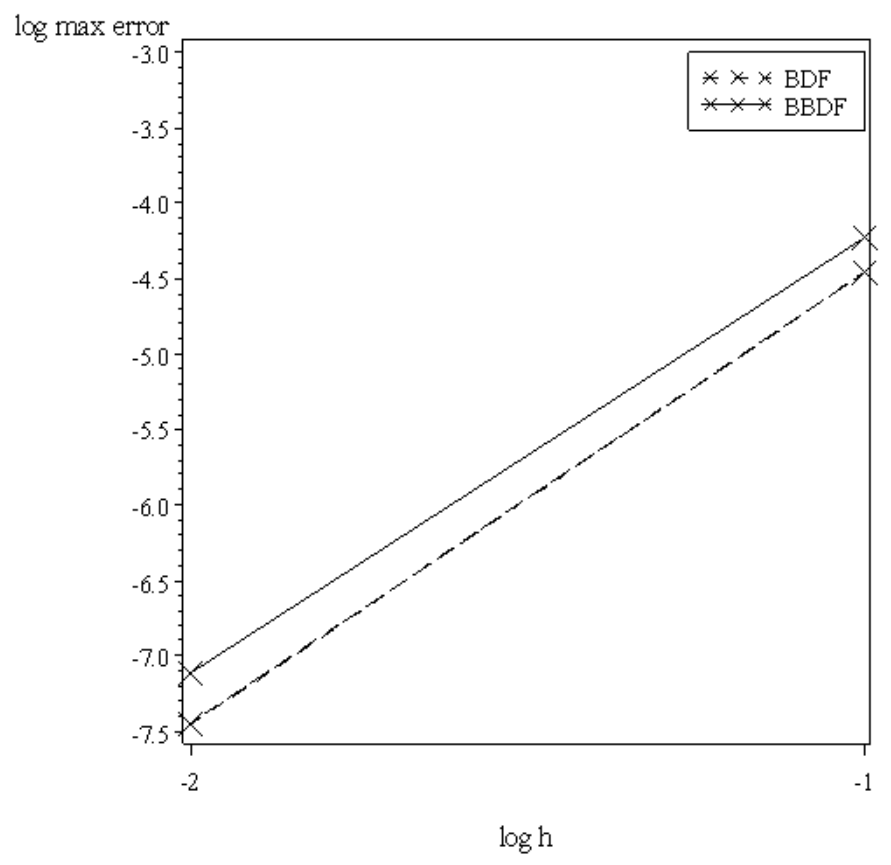

Figure 2: Error of BDF and BBDF at $r=1$ with different step sizes 
Example 2 Consider the initial problem

$$
\begin{aligned}
& y^{\prime \prime}(t)=-y(t)+t, t \geq 0 \\
& y^{\prime}(0)=[1.8+0.2 r, 2.2-0.2 r] .
\end{aligned}
$$

Exact solution at $t=1$ using (1)-differentiability is given by:

$$
\begin{aligned}
& y 1(t ; r)=\left(\frac{4}{5}+\frac{1}{5} r\right) \sin (t)+\left(\frac{9}{10}+\frac{1}{10} r\right) \cos (t)+t \\
& y 2(t ; r)=\left(\frac{6}{5}-\frac{1}{5} r\right) \sin (t)+\left(\frac{11}{10}-\frac{1}{10} r\right) \cos (t)+t .
\end{aligned}
$$

\begin{tabular}{|c|c|c|c|c|c|}
\hline & & \multicolumn{2}{|c|}{$\mathrm{BDF}$} & \multicolumn{2}{|c|}{$\mathrm{BBDF}$} \\
\hline$h$ & $r$ & $\underline{\varepsilon}$ & $\bar{\varepsilon}$ & $\underline{\varepsilon}$ & $\bar{\varepsilon}$ \\
\hline \multirow{6}{*}{$10^{-1}$} & 0 & $1.708944 \mathrm{e}-05$ & $2.85313 \mathrm{e}-05$ & $2.25608 \mathrm{e}-05$ & $4.09196 \mathrm{e}-05$ \\
\hline & 0.2 & $1.823363 \mathrm{e}-05$ & $2.73871 \mathrm{e}-05$ & $2.43967 \mathrm{e}-05$ & $3.90838 \mathrm{e}-05$ \\
\hline & 0.4 & $1.937782 \mathrm{e}-05$ & $2.62430 \mathrm{e}-05$ & $2.62326 \mathrm{e}-05$ & $3.72479 \mathrm{e}-05$ \\
\hline & 0.6 & $2.052201 \mathrm{e}-05$ & $2.50988 \mathrm{e}-05$ & $2.80684 \mathrm{e}-05$ & $3.54120 \mathrm{e}-05$ \\
\hline & 0.8 & $2.166619 \mathrm{e}-05$ & $2.39546 \mathrm{e}-05$ & $2.99043 \mathrm{e}-05$ & $3.35761 \mathrm{e}-05$ \\
\hline & 1.0 & $2.281038 \mathrm{e}-05$ & $2.28104 \mathrm{e}-05$ & $3.17402 \mathrm{e}-05$ & $3.17402 \mathrm{e}-05$ \\
\hline \multicolumn{2}{|c|}{ Execution time } & \multicolumn{2}{|c|}{1.47} & \\
\hline
\end{tabular}

Source: Rabiei et al. [23].

Table 2: Error at $t=1$ in solving problem 2

\begin{tabular}{|c|c|c|c|c|c|}
\hline \multicolumn{2}{|c|}{} & \multicolumn{2}{c|}{ BDF } & \multicolumn{2}{c|}{ BBDF } \\
\hline$h$ & $r$ & $\underline{\varepsilon}$ & $\bar{\varepsilon}$ & $\underline{\varepsilon}$ & $\bar{\varepsilon}$ \\
\hline \multirow{4}{*}{$10-2$} & 0 & $1.67951 \mathrm{e}-08$ & $2.83038 \mathrm{e}-08$ & $3.56459 \mathrm{e}-08$ & $6.04823 \mathrm{e}-08$ \\
\cline { 2 - 6 } & 0.2 & $1.79460 \mathrm{e}-08$ & $2.71529 \mathrm{e}-08$ & $3.81297 \mathrm{e}-08$ & $5.79987 \mathrm{e}-08$ \\
\cline { 2 - 6 } & 0.4 & $1.90969 \mathrm{e}-08$ & $2.60021 \mathrm{e}-08$ & $4.06131 \mathrm{e}-08$ & $5.55149 \mathrm{e}-08$ \\
\cline { 2 - 6 } & 0.6 & $2.02477 \mathrm{e}-08$ & $2.48512 \mathrm{e}-08$ & $4.30966 \mathrm{e}-08$ & $5.30309 \mathrm{e}-08$ \\
\cline { 2 - 6 } & 0.8 & $2.13986 \mathrm{e}-08$ & $2.37003 \mathrm{e}-08$ & $4.55803 \mathrm{e}-08$ & $5.05478 \mathrm{e}-08$ \\
\cline { 2 - 6 } & 1.0 & $2.25495 \mathrm{e}-08$ & $2.25495 \mathrm{e}-08$ & $4.80643 \mathrm{e}-08$ & $4.80643 \mathrm{e}-08$ \\
\hline \multicolumn{2}{|c|}{$2.59 \mathrm{~s}$} & \multicolumn{3}{c|}{$0.12 \mathrm{~s}$} \\
\hline
\end{tabular}

Both methods used in this session solve second order fuzzy differential equations directly without reducing to a system of first order fuzzy differential equations. For problem 1-2, the errors of BDF and BBDF compared with exact solution for $y$ and $\bar{y}$ are given in Table $1-2$. Time taken for the methods to compute the numerical solution is also given in table 1-2.In Table 1-2, we can see that the absolute errors for both methods are very small. Even though the absolute errors obtained by BDF is slightly smaller than the absolute errors obatained by BBDF at different step sizes. However, for the time taken to calculate the results (see Table 1-2), the method proposed in this paper has significant advantages. It is clearly shown that $\mathrm{BBDF}$ is almost as accurate as BDF but BBDF with low computation cost; hence, it is computationally more effiecient. Figure 1 and Figure 3, show the approximate solutions 


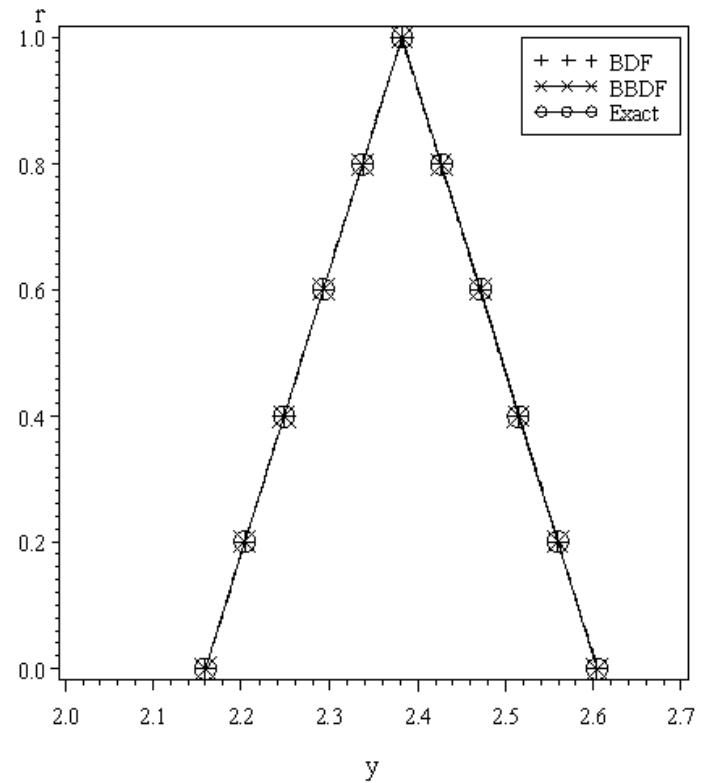

Figure 3: The exact solutions and the approximate solutions in Table 2 with $h=0.1$

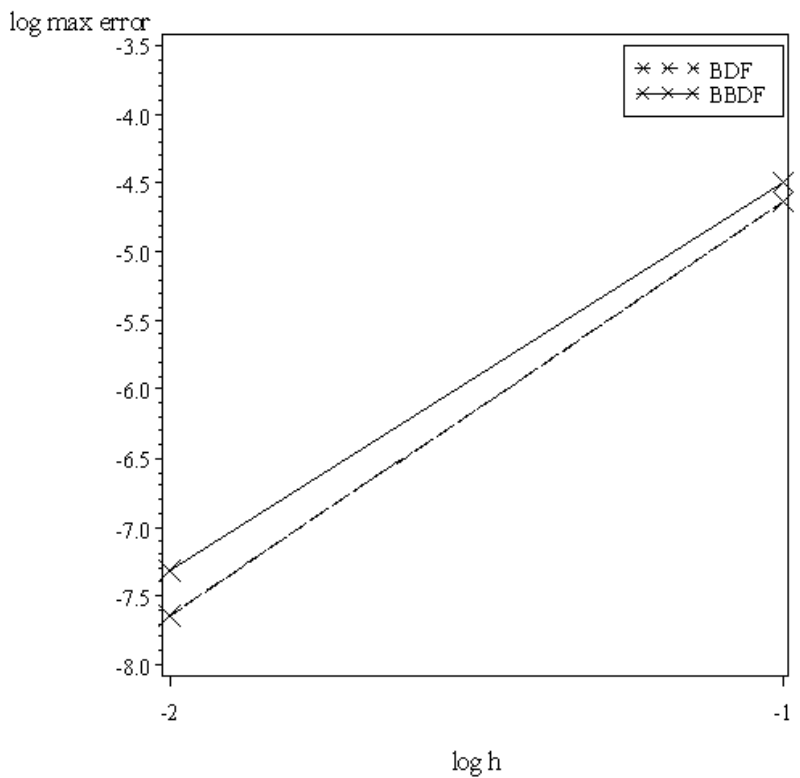

Figure 4: Error of BDF and BBDF at $r=1$ with different step sizes 
of BDF and BBDF are close to exact solution. Figure 2 and Figure 4, show the errors obtained by BDF is smaller than BBDF at different step sizes. In general, BDF are slightly outperform than BBDF in term of the accuracy of the results obtained, whereas BBDF is better than the BDF in term of execution time at different step sizes.

\section{Conclusion}

In this study, we have presented a fuzzy version of block backward differentiation formulas for the solutions of second-order Fuzzy Differential Equations under generalized differentiability. We observe that the value of errors between exact and approximation gets smaller when the step size $h$ is reduced from 0.1 to 0.01 for both methods of BDF and BBDF. After comparing the time taken by the methods, it seems the fuzzy version of block backward differentiation formula is one of the efficient methods for solving second-order fuzzy differential equations in term of the execution time.

The clear advantage of BBDF method is the low computation cost. This is because in BBDF method, two solution i.e. $\underline{y}_{n+1}$ and $\underline{y}_{n+2}$ values are computed simultaneously as discussed in earlier session. This again will lead to a quicker execution time. Therefore, we can conclude that the BBDF method with high accuracy and less execution time compared with the existing BDF method is more efficient for solving second order fuzzy differential equations.

As a future work, we will extend numerical methods for solving second order stiff fuzzy differential equations.

\section{Acknowledgments}

We thank Institute for Mathematical Research (INSPEM) and the Department of Mathematics, Universiti Putra Malaysia for the financial support under Fundamental Grant Scheme (FRGS) 02-02-13-1380FR.

\section{References}

[1] Chang, S. L. and Zadeh, L.A. On fuzzy mapping and control. IEEE Transactions on Systems Man Cybernetics. 1972. 2: 330-340.

[2] Dubois, D. and Prade, H. Towards fuzzy differential calculus part 3: differentiation. Fuzzy Sets and Systems. 1982. 8(3): 225-233.

[3] Puri M.L. and Ralescu, D. Differential and fuzzy functions. Journal of Mathematical Analysis and Applications. 1983. 91(2): 552-558.

[4] Kaleva, O. Fuzzy differential equations. Fuzzy Set System. 1987. 24(3): 301-317.

[5] Kaleva, O. The Cauchy problem for fuzzy differential equations. Fuzzy Sets and Systems. 1990. 35(3): 389-396.

[6] Seikkala, S. On the fuzzy initial value problem. Fuzzy Sets and Systems. 1987.24(3): 319-330. 
[7] Abbasbandy S. and Viranloo, T. Numerical solution of fuzzy differential equation, Mathematical \& Computational Applications. 2002. 7(1): 41-52.

[8] Abbasbandy, S. Viranloo, T. López-Pouso, Ó. and Nieto, J. Numerical methods for fuzzy differential inclusions. Computers $\&$ S Mathematics with Applications. 2004.48(1011): $1633-1641$.

[9] Allahviranloo, T. Ahmady, N. and Ahmady, E. Numerical solution of fuzzy differential equations by predictor-corrector method. Information Sciences. 2007. 177(7): 16331647 .

[10] Allahviranloo, T. Ahmady, E. and Ahmady, N. $n$ th-order fuzzy linear differential equations. Information Sciences. 2008. 178(5): 1309-1324.

[11] Wang, L. and Guo, S. Adomian method for second-order fuzzy differential equation, World Academic of Science. Engineering and Technology. 2011.5: 4-23.

[12] Rabiei, F. Ismail, F. Ahmadian, A. and Salahshour, S. Numerical solution of secondorder fuzzy differential equation using improved runge-kuttanystrom method. Mathematical Problems in Engineering. 2013. 1-10.

[13] Goetschel, R. and Voxman, W. Elementary fuzzy calculus. Fuzzy Sets and Systems. 2013. 18(1): 31-43.

[14] Zimmermann, H. Fuzzy set theory and its applications. Dordrecht: Kluwer Academic Publishers. 1991.

[15] Zimmermann, H. Fuzzy set theory and its applications. Boston, Mass.: Kluwer Academic Publishers. 1992.

[16] Bede, B. and Gal, S. G. Generalizations of the differentiability of fuzzy-number-valued functions with applications to fuzzy differential equations. Fuzzy Sets and Systems. 2005. 151(3): 581-599.

[17] Chalco-Cano, Y. and Román-Flores, H. On new solutions of fuzzy differential equations, Chaos. Solitons $\&$ Fractals. 2008. 38(1): 112-119.

[18] Khastan, A. Bahrami, F. and Ivaz, K. New results on multiple solutions for ??thorder fuzzy differential equations under generalized differentiability. Boundary Value Problems. 2009. 2009: 1-13.

[19] Ibrahim, Z. B. Suleiman, M. and Othman, K. I. Fixed coefficient block backward differentiation formulas for the numerical solution of stiff ordinary differential equations. European Journal of Scientific Research. 2008. 21(3): 508-520.

[20] Ibrahim, Z. B. Suleiman, M. and Othman, K. I. Direct block backward differentiation formulas for solving second order ordinary differential equations. Proceedings of World Academy of Science, Engineering and Technology. 2008. 42: 57.

[21] Rabiei, F., Ismail, F., Ahmadian, A. and Salahshour, S. Numerical solution of secondOrder fuzzy differential equation using improved Runge-Kutta Nystrom method. Mathematical Problems in Engineering, 2013. 1-10. doi:10.1155/2013/803462. 